Aus dem Pharmakologischen Institut der Universität in Zürich. (Direktor: Prof. Dr. Cloetta.)

\section{Ueber die Behandlung der Kreislaufstörung bei Diphtherievergiftung.}

\section{Von A. Iwanowa.}

Die Kreislaufstörungen bei der Diphtherievergiftung sind eine klịnisch nur zul oft beobachtete und auch anatomisch und experimentell mehrfach studierte Erscheinung. Wir haben im Diphtherietoxin ein schweres Zellgift zu sehen, das eine ganze Reihe von Organen im Körper ergreift. So sind bekanntlich besonders hochgradige Leberveränderungen beobachtet, dann gehören Nieren- und Nebennierenläsionen zum anatomischen Bild und ebenso Veränderungen am Herzmuskel. Diese letzteren scheinen sich verschieden zu gestalten, je nach der Jauer der Vergiftung, was ja auch nichts Besonderes auf sich hat. In schweren Fällen kann es zu einer vollständigen Zerstörung des Muskelprotoplasmas kommen.

Mollard und Regaud') haben hieruber sehr ausführliche Studien an Hunden ausgeführt. Die Läsion der Muskelfasern ist bei schwerer akuter Vergiftung diffus, bei der subakuten mehr herdweise. Die diffuse Erkrankung besteht anatomisch in parenchymatöser Trübung mit fettiger Degeneration, bei der subakuten in einer ganzen Reihe verschiedenartiger Veränderungen, die schließßlich zu völliger Vernichtung der Muskelfasern an einzelnen Herden führen können. Daß̧ liese ausgesprochenen Veränderungen in Verbindung mit den Läsionen, die das Gift auch an anderen Organen hervorruft, zu einer schweren Beeinträchtigung der Zirkulation führen können, ist nicht weiter zu verwundern. Es läßt sich denn auch experimentell sehr leicht das starke Sinken des Blutdruckes an mit Diphtherietoxin vergifteten Tieren nachweisen. Hunde und Kaninchen verhalten sich dabei verschieden, indem die ersteren auf große Dosen schon innerhalb weniger Stunden eine Zirkulations. schädigung aufweisen, während beim Kaninchen selbst nach der zwanzigfachen letalen Dosis ein Latenzstadium von etwa 20 Stunden besteht, während welcher keine bemerkbaren Veränderungen sich abspielen. - Worauf beruht nun die gewöhnliche Form der Zirkulationsstörung? (Von der ganz schweren Vergiftung sehe ich hier ab.) Handelt es sich mehr um eine Lähmung der Gefälße oder les Herzens? Die Beantwortung dieser Frage hat auch eine therapeutische Bedentung; denn ja nach ihrem Ausfall wird man, eine vernünftige und pathologisch denkende Therapie vorausgesetzt, die entsprechenden Mittel auszuwählen haben. Romberg und Pässler²) fanden das Vasomotorenzentrum durch reflektorische Erregung nicht mehr beeinflußbar, dagegen konnten sie durch Bauchmassage, $d . h$. durch Kuführen einer großen Blutmenge zum Herzen den Aorten druck steigern. Sie schließen daraus, dab die Herzkrait noch ordentlich erhalten und daß die Blutdrucksenkung hauptsächlich durch Vasomotorenlähmung bedingt sei. Auch Enriquez und $\mathrm{Halli} \mathrm{n}^{8}$ ) fanden eine starke Lähmung des Vasomotorenzentrums; bei Kompression der Aorta sahen sie den Druck ansteigen, aber nicht sehr hoch; sie schließ3en daraus, daß sowohl das Vasomotoren zentrum als auch das Herz gelähmt sei. v. Steyskal') hat den Druck in der Carotis und im linken Vorhof gemessen und für diesen Quotienten der Herzarbeit" ein Sinken bei schwerer Diphtherie"vergiftung der Hunde beobachtet, woraus er anch den Schlul3 zieht, daß beide Teile des Zirkulationsapparates Herz und Gefäße geschädigt seien.

Auch Rolly hat am isolierten Säugetierherz die Schädigung durch Diphtheriegift nachweisen können, daneben hebt er aber auch die intensive Gefäßlähmung hervor. Ob diese letztere Lähunung vielleicht bedingt sei durch eine funktionelle Schädigung der Nebenniere und Inufizienz ihrer Sekretion, wodurch Gefäßdilata-

l) Lésions chroniques du nyocarde consécutives à l'intoxication diphtherięue. Journ. de physiol. et de path. gén. 1899 .

Dentsches Archiv für klinische Medizin Bd. 6

3) Arch. de physiol et de pathol. gén. 1898 .

4) Zeitschrift fitir kliuische Medizin Bd. 44 11. $5 \mathrm{l}$.

5) Archiv für experimenteile Patlologle und Pliarmakologie Bd. $\mathrm{d}_{2}$ tion hervorgerufen werden köunte, hat neulich Ehrmann ${ }^{1}$ ) untersucht; es lief sich jedoch auch bei schwerer Vergiftung keine Verminderung der Adrenalinbildung nachweisen.

Im großen und ganzen scheinen also die experimentellen Arbeiten zunächst sicher eine Lähmung des Vasomotorenzentrums festzustellen, und daneben ist auch eine mit der Dauer der Vergiftung zunehmende Herzschwäche nachgewiesen. Inwieweit diese Störungen maßgebend sind für den letalen Ausgang, soll hier zunächst nicht untersucht werden; die Fragen, die ich mir stellte, waren folgende: 1. Welche Mittel erscheinen geeignet, das erwähnte Symptom der Diphtherievergiftung zu bekämpfen, und 2. läßt sich durch systematische Anwendung eines als wirksam befundenen Mittels der Verlauf der Vergiftung beinflussen?

Als Versuchstiere dienten ausschließlich Kaninchen; das Diphtheriegift wurde uns in reichlicher Menge zuvorkommender Weise von Herrn Prof. Kolle in Bern zur Verfügung gestellt. Ich orientierte mich zunächst über die Giftigkeit des Präparats, indem ich verschiedenen Tieren steigende Mengen einspritzte. Es zeigte sich, daf man mit ziemlicher Genauigkeit den Verlauf der Vergiftung bestimmen kann. Wurde einem Tier 0,1 ccm per kg eingespritzt, so trat der Tod unter schweren Lähmungserscheinungen schon nach etwa 30 Stunden ein; wurden dagegen nur $0.025 \mathrm{ccm}$ pro $\mathrm{kg}$ injiziert, so dauerte die Vergiftung vier bis fünf Tage und verlief dabei stets in charakteristischer Weise. Es wurde bei den Kaninchen sehr darauf geachtet, stets ganz gleichmäBiges Tiermaterial aus derselben Zucht zu verwenden und diesem Umstande ist es auch zu danken, daß ich mit ziemlicher Sicherheit je nach der Dosis den Verlauf bestimmen konnte. Wurden $0,025 \mathrm{ccm}$ gebraucht, so erguben sich folgende typische Veränderungen, die ich an einem Beispiele anführe.

Normal. Nach der lnjektion.

\begin{tabular}{|c|c|c|c|c|c|c|}
\hline & & 1. Tag & 2. Tag & 3. Tag & 4. Tag & 5. Tag \\
\hline $\begin{array}{l}\text { Gewicht : } \\
\text { Puls : } \\
\text { Atmung : } \\
\text { Temperatur: }\end{array}$ & $\begin{array}{r}3000 \\
240 \\
180 \\
39,7\end{array}$ & $\begin{array}{r}2900 \\
280 \\
128 \\
40,0\end{array}$ & $\begin{array}{r}2850 \\
280 \\
140 \\
39,4\end{array}$ & $\begin{array}{r}2800 \\
260 \\
80 \\
38,0\end{array}$ & $\begin{array}{r}2750 \\
240 \\
120 \\
37,2\end{array}$ & Tod \\
\hline
\end{tabular}

Wie ersichtlich, nimmt das Köpergewicht vom ersten bis zweiten Tage an ab, die Temperatur stieg in einigen Fällen am ersten Tage, von da ab beginnt sie langsam zu sinken; stärkeres Fieber habe ich nie beobachtet, eine ganz interessante Erscheinung fitr die Lehre der Toxinwirkung. Die Atmung reduzierte sich auch parallel mit dem Sinken der Temperatur. Die Pulszahl zeigte unreglmäßiges Verhalten, bald stieg sie etwas, bald nahm sie eher ab. Stets traten gegen das Ende hin typische Lähmungen der hinteren Extremitäten auf. Man hatte den Eindruck, dab es sich hinteren aller Funktionen handelt. Nachdem so die allgemeine Wirkung und der Verlauf festgestellt waren, begann ich mit dem Studium der Zirkulationsstörungen. Es wurde bei einem Kaninchen Pulskurve und Blutdruck aufgezeichnet, dann das Tier mit Diphtherietoxin injiziert und je nach der Höhe der Dosis nach 30 oder 48 Stunden der Blutdruck wieder untersucht. Er zeigte sich stets erheblich verändert, und in diesem pathologischen Stadium wurden dann die therapeutischen Experimente durchgeführt, tiber die ich jetzt ausführlich berichten will. Sämtliche Tiere erhielten vor jedem Blutdruckversuch $1 \mathrm{~g}$ Urethan subkutan zur allgemeinen Beruhigung. Alle medikamentösen Injektionen wurden intravenös ge macht. Bei der Blutdruckmessung wurde am gedämpften $\mathrm{Hg}-\mathrm{Mano}$ meter der mittlere Druck bestimmt und mit dem $\mathrm{H}$ ïrthleschen Tonometer die Pulsamplitude gemessen.

Versuch Kaninchen No. 8.

\begin{tabular}{|c|c|c|c|c|c|}
\hline Zeit & $\begin{array}{l}\text { Blut- } \\
\text { druck } \\
\mathrm{nmg} \mathrm{Hg}\end{array}$ & $\begin{array}{l}\text { Puls- } \\
\text { zahl }\end{array}$ & Amplitude & $\begin{array}{l}\text { Ge- } \\
\text { wicht } \\
g\end{array}$ & Bemerkungen \\
\hline $\begin{array}{l}\text { 1. Juni } \\
10 \text { Uhr morgens }\end{array}$ & 104 & 240 & $4 \mathrm{mnl}$ & 2300 & $\begin{array}{l}\text { erhält } 0,04 \mathrm{ccm} \text { DT pro } \mathrm{kg} \text { sub- } \\
\text { kutan. }\end{array}$ \\
\hline $\begin{array}{l}\text { 3. Juni } \\
4 \\
4,5\end{array}$ & $\begin{array}{l}80 \\
70\end{array}$ & 246 & $11 / \mathrm{s} \min$ & 2000 & $\begin{array}{l}\text { sehr geringe Amplitude, } \\
\text { s. Kurve } 1 \text {. }\end{array}$ \\
\hline $\begin{array}{l}4,7 \\
4,9 \\
4,12 \\
4,15\end{array}$ & $\begin{array}{r}130 \\
70\end{array}$ & 308 & $3 y / 211101$ & & $\begin{array}{l}\text { lujektion von } 0,02 \text { Coffein. } \\
\text { Injektion } 0,02 \text { Coffein und Di- } \\
\text { galen } 2 \text { ccin. }\end{array}$ \\
\hline $\begin{array}{l}4,17 \\
-1,20\end{array}$ & $\begin{array}{l}130 \\
125\end{array}$ & 252 & $24: 2 n$ & & $\begin{array}{l}\text { In der Kurve Il tritt die ver- } \\
\text { besserte GefäBspannung } \\
\text { deutlich hervor. }\end{array}$ \\
\hline $4,3 i$ & 110 & 261 & & & \\
\hline
\end{tabular}

1) Archiv tïr experimentelle Pathologie und Pharnakologic Bd, is 

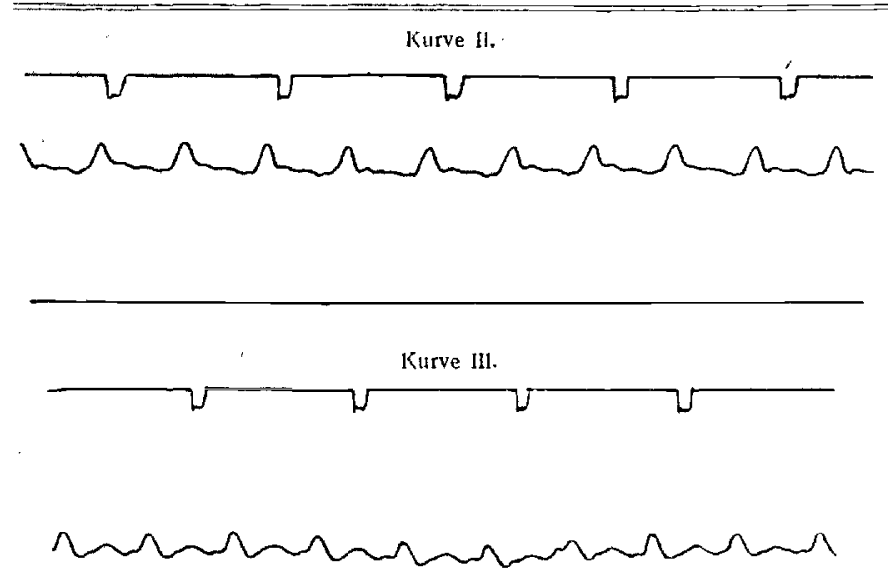

Kurve IV,

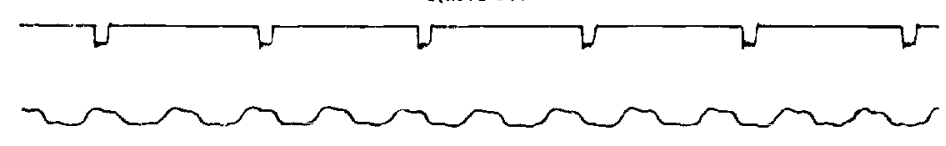

Kurve ].
Menschen festgestellt, daB die Kombination von Coffein und Digalen sehr günstige Resultate ergibt, was auch noch an zwei weiteren Vorsuchen festgestellt wurde.

Am moisten gespannt war ich auf die Wirkung des Kampfers, wird er doch immer noch auch bei Diphtherie als das Primum refugium bei Herzschwäche betrachtet, obwohl ja gerade das spasmophile Alter der Patienten für einen ausgiebigen Gebrauch dieses Mittels manchmal Bedenken hat.

Versuch Kaninchen E.

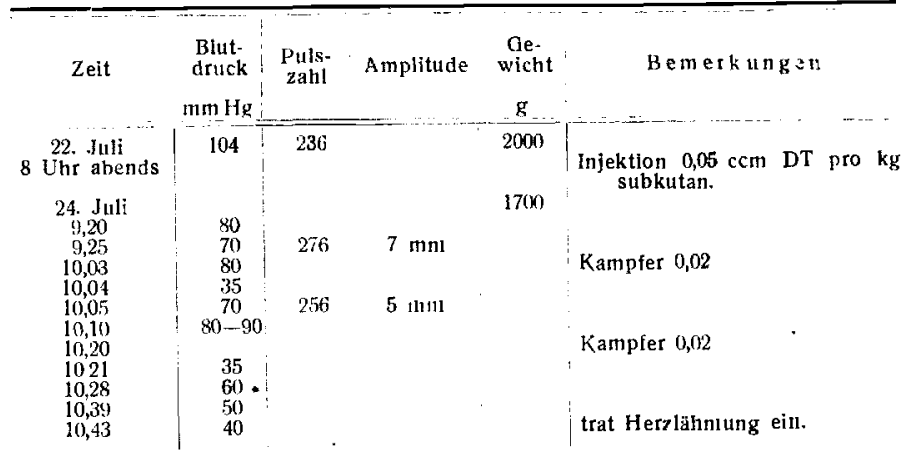

In ganz gleicher Weise verliefen auch zwei weitere Kampferversuche. Die Resultate haben mich enttäuscht; ich hatte gehofft, bei dieser Infektionskrankheit noch einen günstigen Einfluß des Kampfers feststellen zu können. Steht dieses Resultat im Gegensauz zur klinischen Beobachtung? Das ist schwer zu sagen, weil einwandfreie Untersichungen hierüber speziell nicht vorliegen. Wenn etwa 12-24 Stunden nach Kampferbehandlung der Puls besser wira, so ist damit ja nicht gesagt, $\mathrm{daB}$ dies eine Herzwirkung sei, es kann sich auch um eine Beeinflussung des zentralen Nervensystems handeln, entweder, indein das Atmungszentrum oder, wie vielfach für Kampfer noch angenommen, das Vasomotorenzentrum angeregt wird. Um dieser Sache noch näher zu kommen, habe ich versucht, festzustellen. wie grol3 der Anteil der Gefäße und des Herzens an den Störungen in meinen') Diphtherievergiftungen ist. Z/1 diesem Zweck wurde während des Blutdruckversuchs teils reflektorisch das Gefäbzentrum erregt, teils die Strombahn stark eingeengt durch Kompression der Aorta iber dem Zwerchfell; um dieses letztere leicht und ohne jede Nebenverletzung ausfïhren zu können, wurden aut der linken Thoraxseite drei Rippen reseziert unter sorgfältiger Vermeidung jeder Blıtung, die Aorta angeschlungen Ind im gegebenen Moment der Faden zugezogen. Wird die Operation sorgfältig ausgeführt, so erleidet der Blutdruck dadurch allein nicht die geringste Schwankung.

Puls irregulär, dicrot, s. Kurve IH. Coffein 0,02 .

Digalen $2 \mathrm{ccm}$.

s. Kurve IV, gute Spannung.

lis ergibt sich aus diesen beiden Versuchen, laß Coffein voribergehend den Blutdruck zu steigern vermag, bald aber wieder eine Senkung erfolgt, daß dagegen diese Steigerung viel anhaltender ist, wenn sich zur Coffeinwirkung Digitalis addiert. Im folgenden wurde der Versuch ungekehrt, indein zuerst Digalen gegreben wurde, um den Effekt von diesen allein festzustellen und dann Coffein nachgeschickt.

Versuch Kauinchen C

\begin{tabular}{|c|c|c|c|c|c|}
\hline Zeit & $\begin{array}{l}\text { Blut- } \\
\text { drucli }\end{array}$ & $\begin{array}{l}\text { Puls- } \\
\text { zillut }\end{array}$ & Amplitude & $\begin{array}{c}\text { Ge- } \\
\text { wicht }\end{array}$ & Bemerkungen \\
\hline$\ldots$ & mintlyg & & & $\mathrm{g}$ & \\
\hline $\begin{array}{l}\text { 18. Juli } \\
\text { 10 Uhr moreens }\end{array}$ & 104 & 240 & & 2000 & \\
\hline $19 \mathrm{Ju} 1 \mathrm{i}$ & & & & 1550 & errall $0,040 \mathrm{ccm}$ prer $\mathrm{kg}$. \\
\hline $\begin{array}{r}4,23 \\
4,32 \\
4,40\end{array}$ & $\begin{array}{l}90 \\
80 \\
85\end{array}$ & 254 & $5 \mathrm{~mm}$ & & \\
\hline 1,44 & 110 & 27 & 5111111 & & Digalent $2 \mathrm{ccm}$. \\
\hline 4,51 & lon & & & & Coffein 0,03 intrivetlis. \\
\hline 4,57 & 110 & & & & \\
\hline 5,03 & 165 & & & & \\
\hline 5,05 & 105 & 283 & 4111111 & & \\
\hline 5.15 & 105 & & & & \\
\hline
\end{tabular}

Fis craab sich dabei eine krätige und anhaltende Steigr'tung durch Digilen allein: diese wurde aber noch weiter gefördert und namontich stabilor gemacht durch die nachfolsende Coffeindose; irh kann alse nur bestätigen was bereits Doctoruwitsch') an!

1) Dortorowitsch, Therapie der Gegenwarl 19k, S. 343.

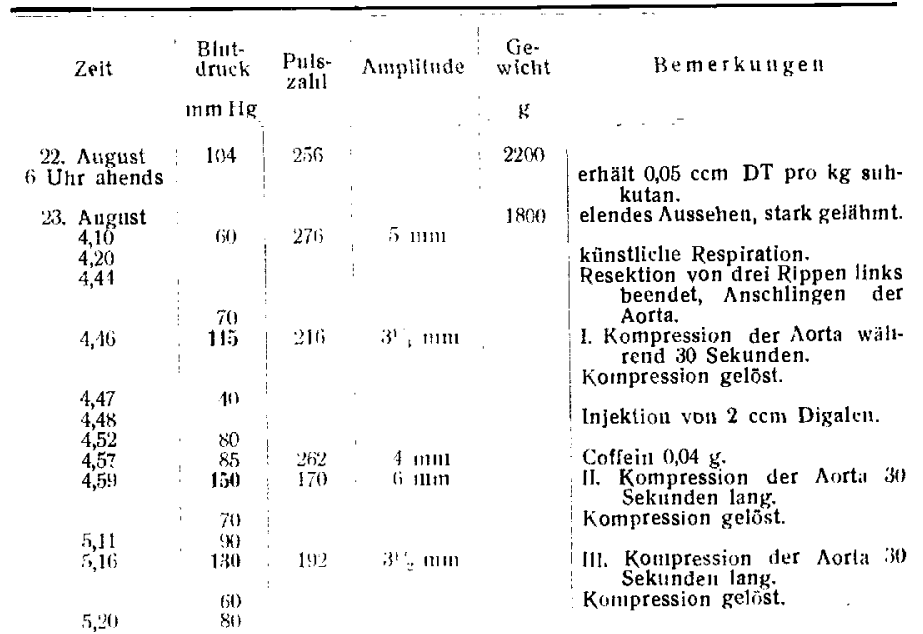

(Fortsetrung der 'Tabelle aluf Seite 2l, oben.)

Nachstehenıl gebe ich noch die zu liesen Vorsischen gehörigen liurven. Die erste entspricht der Erhebung, die der Blitdruck erfälırt nnter Kampfer, sie ist wie ohen ersiclitlich identisch mit, der ohne Kanufer; die zweite, diejenige unter Coffein und Digalen; der Blutdruck ist der mittlere in $1 \mathrm{Ig}$ mm aufgexeichnet von der rifte sich in dieser lichtung velschieden verhalten.
1) Es ist ja von vornherein möglich, dalik verschielrn. Diphtherie 


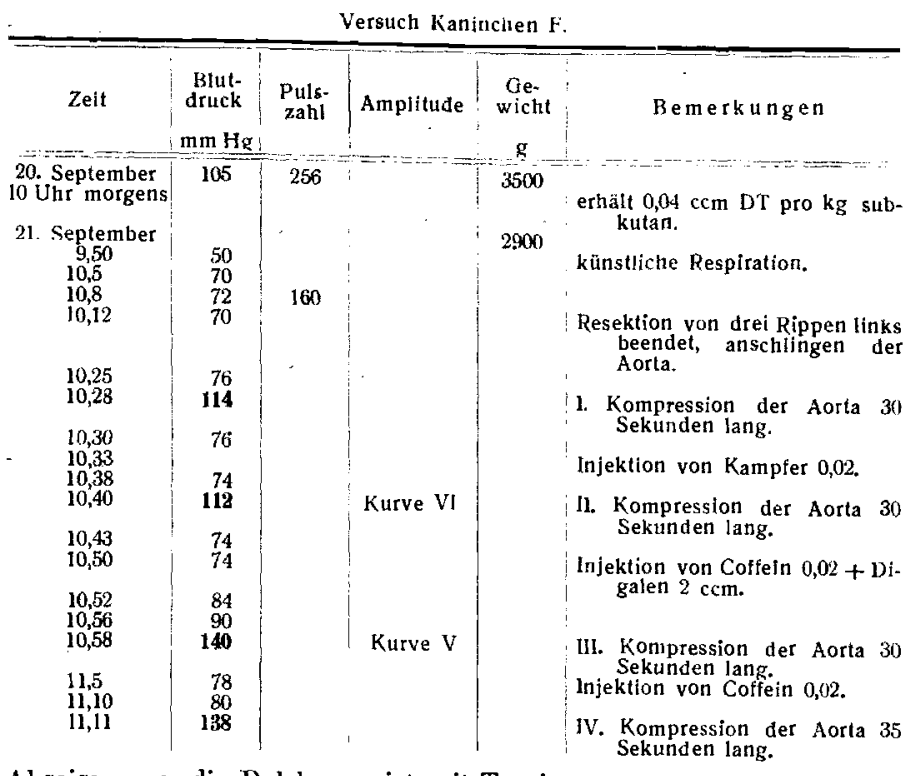

Abscisse aus, die Pulskurve ist mit Torsionsmanoneter geschrieben; Zeitregistrierung $=1 / 2$ Sekunde.

Kurve V.

Kurve vi.

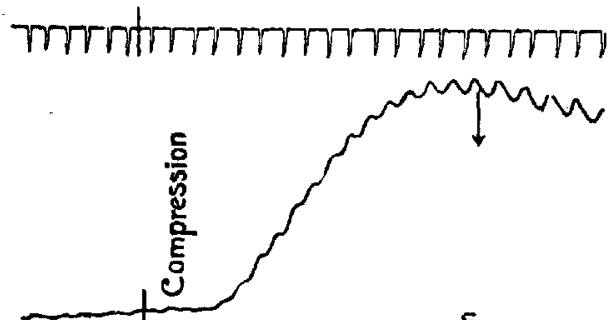

$E$
E
主

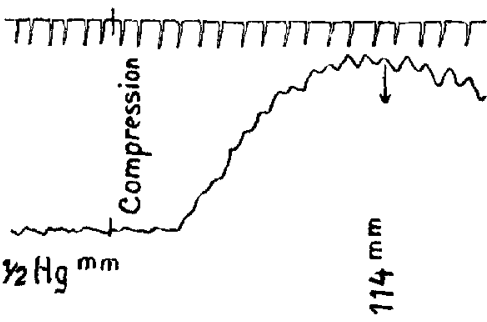

$\pm$

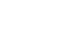
zunähme.

bei erhaltener Zirkulation der Blutdruck steigt und bei Ausschaltung der Mehrzahl der Gefäße die Leistungsfähigkeit des Herzens gegenüber dem erhöhten Widerstande obenfulls relativ und absolut zunimmt. Dieses letztere Experiment beweist, da neben der Erhöhung des Gefäblonus auch die Leistungsfähigkeit des Herzens durch die Therapie steigt, und das ist sehr wichtig, denn durch die Gefäßkontraktion wird dem Herzen mehr Blut, d. h. mehr Arbeit zugeführt, die zu bewältigen vielleicht Schwierigkeiten machen würde, wenn nicht gleichzeitig die Energie des Herzmuskels

Nachdem ich so an Hand der akuten Zirkulationsstörung festgestellt, was für Mittel geeignet sind, diese Störungen zu beseitigen, wandte ich mich dem Studium der zweiten Frage zu, ob durch systematische Verabreichung solcher Mittel sich irgend eine Veränderung im Krankheitsverlauf erzielen lasse.

Es wurden zu diesem Zweck je zwoi Tiere mit den gleichen Mengen Diphtherietoxin eingespritzt und dann eins davon mit dem Medikament behandelt. Bei sämtlichen Tieren wurden Puls und Atmung gezählt, die Temperatur gemessen und das Gowicht festgestellt. Es zeigte sich hier wieder dieselbe konstante Erscheinung der Abnahme des Gewichts und der Körpertemperatur, auch bei den medikamentös behandelten Tieren.

Es wurden behandelt im ganzen: drei Tiere mit Kampfer, sechs mit Coffoin, drei mit Digalen, sechs mit Coffein und Digalen.

Ueber diese Experimente, die im Verlauf von zehn Wochen durchgeführt wurden, kann ich mich kurz fassen, da die Resultate ganz eindeutig sind. Irgend ein Einfluß auf den definitiven Ausgang der Erkrankung hat sich nicht feststellen lassen, sämtliche Tiere, welche die sicher letale Dosis erhalten hatten, sind mit oder ohne Behandlung zugrunde gegangen. Es entspricht dies ja auch der schweren, allgemeinen Intoxikation und Zelldegeneration, die das Diphtheriegift an den verschiedenen Organen hervorruft; ein Verlauf, an dem die vorübergehende Besserung der Zirkulationsverhältnisse offenbar nichts mehr zu ändern vermag. Es hätten sich vielleicht noch feinere Nuancierungen ergeben, wenn man den Tieren die „annähernd“ letale Dosis gegeben hätte; damit wäre aber auch der persönlichen Auslegung und Willkür Tür und Tor geoffinet gewesen.

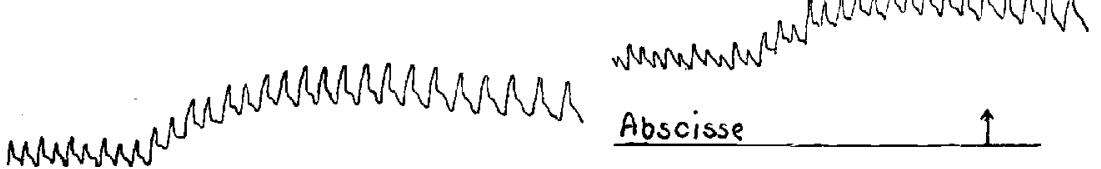

Wir kommen somit auf Grund all des vorliegenden experimentellen Materials zu der Schlublolgerung, dah die einzig rationelle Therapie nur in der Bindung des Gifies durch das Antitoxin bestehen kann, um die allgemeine Zelldegeneration möglichst aufzuhalten. Wenn daneben dann bei einem Individuum sich das Zirkulationssystem besonders leicht affizierbar erweist, so wäre diese isolierte Störung nach den oben entwickelten Anschauungen zu bekämplen und zwar mit um so größerer Aussicht auf Erfolg, je weniger die andern Organe affiziert, $j \theta$ isolierter die Störung am Kreislauf ist. das Herz trotz der schweren Intoxikation noch über ziemliche Reservekräfte verfügt, indem ohne Medikamente auf Aortenkompression eine Steigerung um $34 \mathrm{~mm} \mathrm{Hg}$ zustande kommt; es zeigt sich ferner, daß eine Kampferinjektion an dieser Leistungsfähigkeit nichts ändert (zweite Kompression). Dagegen ruft auch hier die Injektion von Digalen und Coffein eine Druckerhöhung hervor; bei der dritten Kompression steigt der Druck um $50 \mathrm{~mm} \mathrm{Hg}$ absolut und um $26 \mathrm{~mm}$ Hg höher, als während der Kampferwirkung, trotzdem das Herz durch die zwei vorhergehenden Kompressionen schon aufs auBerste angestrengt worden; bei der vierten Kompression stieg der Druck nochmals auf $138 \mathrm{~mm}$. Es wurde dann auch ein Versuch mit faradischer Reizung des Trigeminus und Erstickungsversuch ausgeführt, ohne daß es damit gelang, den Blutdruck wesentlich in die Höho zu treiben. Genau in derselben Weise verliefen noch zwei weitere Experimente unter denselben Bedingungen ausgefúbrt.

A uf Grund aller dieser Ergebnisse komme ich zu folgenden Schlußfolgerungen: Bei der Blutdrucksenkung im Verlauf der Diphtherievergiftung ist in erster Jínie die Gefäblähmung maßgebend, denn das Herz vermag noch erhebliche Widerstände zu überwinden. Kampferdarreichung ist nicht imstande, den Blutdruck zu heben, was leicht begreiflich, da Kampfer eher eine gefäßerweiternde Wirkung hat. Aber auch die Arbeitsleistung des Herzens selber bei Ausschaltung des GroBteils der gelähmten Gefäbe vermag Kampfer nicht zu heben. Dagegen ist entsprechend dem pathologischen Zustande: starke Gefäßlähmung, leichtere Herzlähmung, die Kombination von Digitalis und Coffein sehr wirksam, was sich daraus ergibt, daق 